\title{
A Banach Algebra Similar to Cameron-Storvick's One with Its Equivalent Spaces
}

\author{
Dong Hyun Cho \\ Department of Mathematics, Kyonggi University, Suwon 16227, Republic of Korea \\ Correspondence should be addressed to Dong Hyun Cho; j94385@kyonggi.ac.kr
}

Received 5 February 2018; Revised 6 April 2018; Accepted 16 April 2018; Published 3 June 2018

Academic Editor: Hugo Leiva

Copyright (c) 2018 Dong Hyun Cho. This is an open access article distributed under the Creative Commons Attribution License, which permits unrestricted use, distribution, and reproduction in any medium, provided the original work is properly cited.

Let $C[0, T]$ denote an analogue of a generalized Wiener space, that is, the space of continuous, real-valued functions on the interval $[0, T]$. In this paper, we introduce a Banach algebra on $C[0, T]$ which generalizes Cameron-Storvick's one, the space of generalized Fourier-Stieltjes transforms of the $\mathbb{C}$-valued, and finite Borel measures on $L^{2}[0, T]$. We also investigate properties of the Banach algebra on $C[0, T]$ and equivalence between the Banach algebra and the Fresnel class which plays a significant role in Feynman integration theories and quantum mechanics.

\section{Introduction}

Let $C_{0}[0, T]$ denote the Wiener space, that is, the space of continuous, real-valued functions $x$ on the interval $[0, T]$ with $x(0)=0$. The (generalized) Paley-Wiener-Zygmund (PWZ) stochastic integrals on the (generalized) Wiener space have been used in various papers, in particular, concerning Feynman integration theories [1-4]. In particular, the PWZ integral is used in the definition of Cameron-Storvick's Banach algebra $\mathcal{S}$ of functions on $C_{0}[0, T]$ which is the space of generalized Fourier-Stieltjes transforms of the $\mathbb{C}$-valued and finite Borel measures on $L^{2}[0, T]$ [1]. Johnson [4] showed that $\mathcal{S}$ is isometrically isomorphic to the Banach algebra of the Fresnel integrable functions given by Albeverio and Høegh-Krohn [5]. Further work for relationships between the Banach algebra $\delta$ and the Fresnel class was studied by Chang et al. [6] on infinite dimensional Hilbert spaces, and the same work was done by Chang et al. [7] on the space $C_{\alpha, \beta}[0, T]$ which is a generalized Wiener space with mean function $\alpha$ and variance function $\beta$, where $\alpha$ and $\beta$ are appropriate functions on $[0, T]$. We note that every Wiener path $x$ in both $C_{0}[0, T]$ and $C_{\alpha, \beta}[0, T]$ starts at the origin; that is, $x(0)=0$.

On the other hand, let $C[0, T]$ denote an analogue of a generalized Wiener space, that is, the space of continuous, real-valued functions on the interval $[0, T]$. On the space $C[0, T]$, Ryu $[8,9]$ introduced a finite measure $w_{\alpha, \beta ; \varphi}$ and investigated its properties, where $\alpha, \beta:[0, T] \rightarrow \mathbb{R}$ are continuous functions such that $\beta$ is strictly increasing, and $\varphi$ is arbitrary finite measure on the Borel class $\mathscr{B}(\mathbb{R})$ of $\mathbb{R}$. On this space $\left(C[0, T], w_{\alpha, \beta ; \varphi}\right)$, the author [10] introduced an Itô type integral $I_{\alpha, \beta}$ and a generalized PWZ integral with their relation. The relation says that $I_{\alpha, \beta}$ is reduced to the generalized PWZ integral if $\varphi$ is a probability measure on $\mathscr{B}(\mathbb{R})$ and the generalized PWZ integral exists. In this paper, we will introduce a Banach algebra $\mathcal{S}_{\alpha, \beta ; \varphi}$ on $C[0, T]$ by using $I_{\alpha, \beta}$, which generalizes Cameron-Storvick's Banach algebra $\mathcal{S}$ with the mean function and the variance function determined by $\alpha$ and $\beta$, respectively. We also investigate properties of $\mathcal{S}_{\alpha, \beta ; \varphi}$, and relationships between $\mathcal{S}_{\alpha, \beta ; \varphi}$ and the Fresnel class [5] which plays a significant role in Feynman integration theories and quantum mechanics. We note that every path in $C[0, T]$ starts at arbitrary point so that $C[0, T]$ generalizes both $C_{0}[0, T]$ and $C_{\alpha, \beta}[0, T]$.

When the generalized PWZ integral on $C[0, T]$ is defined, one of difficulties encountered is the existence of a complete orthonormal basis of functions in $L_{\alpha, \beta}^{2}[0, T]$ such that these functions are of bounded variation and orthogonal in $L_{0, \beta}^{2}[0, T]$, where $L_{0, \beta}^{2}[0, T]$ and $L_{\alpha, \beta}^{2}[0, T]$ are the $L^{2}$-spaces with respect to the Lebesgue-Stieltjes measures induced by $\alpha$ and $\beta$ [10]. In order to avoid this difficulty, we will use $I_{\alpha, \beta}$ instead of the generalized PWZ integral so that we can define the functions in $\mathcal{S}_{\alpha, \beta ; \varphi}$ regardless of the existence of the 
orthonormal basis of $L_{\alpha, \beta}^{2}[0, T]$ satisfying the orthogonality in $L_{0, \beta}^{2}[0, T]$ as described above.

\section{An Analogue of a Generalized Wiener Space}

In this section, we introduce an analogue of a generalized Wiener space with the Itô type integral as described in Section 1.

Let $m_{L}$ denote the Lebesgue measure on $\mathbb{R}$. Let $C[0, T]$ denote the space of continuous, real-valued functions on the interval $[0, T]$. Let $\alpha, \beta:[0, T] \rightarrow \mathbb{R}$ be two continuous functions, where $\beta$ is strictly increasing. Let $\varphi$ be a positive finite measure on $\mathscr{B}(\mathbb{R})$. For $\vec{t}_{n}=\left(t_{0}, t_{1}, \ldots, t_{n}\right)$ with $0=$ $t_{0}<t_{1}<\cdots<t_{n} \leq T$, let $J_{\vec{t}_{n}}: C[0, T] \rightarrow \mathbb{R}^{n+1}$ be the function given by

$$
J_{\vec{t}_{n}}(x)=\left(x\left(t_{0}\right), x\left(t_{1}\right), \ldots, x\left(t_{n}\right)\right) .
$$

For a rectangle $\prod_{j=0}^{n} B_{j}$ in $\mathscr{B}\left(\mathbb{R}^{n+1}\right)$, the subset $J_{\vec{t}_{n}}^{-1}\left(\prod_{j=0}^{n} B_{j}\right)$ of $C[0, T]$ is called an interval $I$ and let $\mathscr{C}$ be the set of all such intervals $I$. Define a premeasure $m_{\alpha, \beta ; \varphi}$ on $\mathscr{C}$ by

$$
\begin{aligned}
& m_{\alpha, \beta ; \varphi}(I)=\int_{B_{0}} \int_{\prod_{j=1}^{n} B_{j}} W_{n}\left(\alpha, \beta, \vec{t}_{n}, \vec{u}_{n},\right. \\
& \left.u_{0}\right) d m_{L}^{n}\left(\vec{u}_{n}\right) d \varphi\left(u_{0}\right),
\end{aligned}
$$

where for $\vec{u}_{n}=\left(u_{1}, \ldots, u_{n}\right) \in \mathbb{R}^{n}$ and $u_{0} \in \mathbb{R}$,

$$
\begin{aligned}
W_{n}\left(\alpha, \beta, \vec{t}_{n}, \vec{u}_{n}, u_{0}\right) \\
=\left[\frac{1}{\prod_{j=1}^{n} 2 \pi\left[\beta\left(t_{j}\right)-\beta\left(t_{j-1}\right)\right]}\right]^{1 / 2} \\
\quad \times \exp \left\{-\frac{1}{2} \sum_{j=1}^{n} \frac{\left[u_{j}-\alpha\left(t_{j}\right)-u_{j-1}+\alpha\left(t_{j-1}\right)\right]^{2}}{\beta\left(t_{j}\right)-\beta\left(t_{j-1}\right)}\right\} .
\end{aligned}
$$

The Borel $\sigma$-algebra $\mathscr{B}(C[0, T])$ of $C[0, T]$ with the supremum norm coincides with the smallest $\sigma$-algebra generated by $\mathscr{C}$ and there exists a unique positive finite measure $w_{\alpha, \beta ; \varphi}$ on $\mathscr{B}(C[0, T])$ with $w_{\alpha, \beta ; \varphi}(I)=m_{\alpha, \beta ; \varphi}(I)$ for all $I \in \mathscr{C}$. This measure $w_{\alpha, \beta ; \varphi}$ is called an analogue of a generalized Wiener measure on $(C[0, T], \mathscr{B}(C[0, T]))$ according to $\varphi[8,9]$.

For further work, we give additional conditions for $\alpha$ and $\beta$. Let $\alpha$ and $\beta$ be functions defined on $[0, T]$ such that $\alpha$ is absolutely continuous and $\beta^{\prime}$ is continuous, positive, and bounded away from 0 . We observe that the functions $\alpha$ and $\beta$ induce a Lebesgue-Stieltjes measure $v_{\alpha, \beta}$ on $[0, T]$ by

$$
v_{\alpha, \beta}(E)=v_{\alpha}(E)+v_{\beta}(E),
$$

where $v_{\alpha}(E)=\int_{E} d|\alpha|(t)$ and $v_{\beta}(E)=\int_{E} d \beta(t)$ for a Lebesgue measurable subset $E$ of $[0, T]$. Define $L_{\alpha, \beta}^{2}[0, T]$ to be the space of functions on $[0, T]$ that are square integrable with respect to the measure $v_{\alpha, \beta}$; that is,

$$
\begin{aligned}
& L_{\alpha, \beta}^{2}[0, T] \\
& \quad=\left\{f:[0, T] \longrightarrow \mathbb{R} \mid \int_{0}^{T}[f(t)]^{2} d v_{\alpha, \beta}(t)<\infty\right\} .
\end{aligned}
$$

The space $L_{\alpha, \beta}^{2}[0, T]$ is in fact a Hilbert space (as our notation suggests) and has the obvious inner product [11]

$$
\langle f, g\rangle_{\alpha, \beta}=\int_{0}^{T} f(t) g(t) d v_{\alpha, \beta}(t)
$$

$$
\text { for } f, g \in L_{\alpha, \beta}^{2}[0, T] \text {. }
$$

Let $S[0, T]$ denote the collection of all step functions on $[0, T]$. For $f$ in $L_{\alpha, \beta}^{2}[0, T]$, let $\left\{\phi_{n}\right\}$ be a sequence of the step functions in $S[0, T]$ with $\lim _{n \rightarrow \infty}\left\|\phi_{n}-f\right\|_{\alpha, \beta}=0$. Define the Itô type integral $I_{\alpha, \beta}(f)$ of $f$ by the $L^{2}(C[0, T])$-limit

$$
I_{\alpha, \beta}(f)(x)=\lim _{n \rightarrow \infty} \int_{0}^{T} \phi_{n}(t) d x(t)
$$

for all $x \in C[0, T]$ for which this limit exists, where $\int_{0}^{T} \phi_{n}(t) d x(t)$ denotes the Riemann-Stieltjes integral of $\phi_{n}$ with respect to $x$. We note that $I_{\alpha, \beta}(f)(x)$ exists for $w_{\alpha, \beta ; \varphi}$ a.e. $x \in C[0, T]$ and the limit in (7) is independent of choice of the sequence $\left\{\phi_{n}\right\}$ in $S[0, T]$. Moreover, one can show that $I_{\alpha, \beta}$ is an injective, bounded linear operator from $L_{\alpha, \beta}^{2}[0, T]$ into $L^{2}(C[0, T])$. The following lemma is due to a result in [10].

Lemma 1. If $f \in L_{\alpha, \beta}^{2}[0, T]$ and $x$ is absolutely continuous on $[0, T]$ with $x^{\prime} \in L_{0, \beta}^{2}[0, T]$, then $\lim _{n \rightarrow \infty} \int_{0}^{T} \phi_{n}(t) d x(t)$ exists and it is given by

$$
\lim _{n \rightarrow \infty} \int_{0}^{T} \phi_{n}(t) d x(t)=\int_{0}^{T} f(t) x^{\prime}(t) d m_{L}(t)
$$

for any sequence $\left\{\phi_{n}\right\}$ of the step functions in $S[0, T]$ with $\lim _{n \rightarrow \infty}\left\|\phi_{n}-f\right\|_{\alpha, \beta}=0$.

For $x \in C[0, T]$ satisfying the assumption in Lemma 1 , we redefine $I_{\alpha, \beta}(f)(x)$ as $I_{\alpha, \beta}(f)(x)=\int_{0}^{T} f(t) x^{\prime}(t) d m_{L}(t)$. Nevertheless, we have (7) for $w_{\alpha, \beta ; \varphi}$ a.e. $x \in C[0, T]$. Also, $I_{\alpha, \beta}$ is still an injective, bounded linear operator from $L_{\alpha, \beta}^{2}[0, T]$ into $L^{2}(C[0, T])$.

Throughout the remainder of this paper, unless otherwise specified, we assume that $\varphi(\mathbb{R})=1$; that is, $\varphi$ is a probability measure on $\mathscr{B}(\mathbb{R})$. We now have the following generalized PWZ theorem [10].

Theorem 2. Let $\left\{f_{1}, \ldots, f_{n}\right\}$ be a set of functions in $L_{\alpha, \beta}^{2}[0, T]$ which are nonzero and orthogonal in $L_{0, \beta}^{2}[0, T]$. Then $I_{\alpha, \beta}\left(f_{1}\right), \ldots, I_{\alpha, \beta}\left(f_{n}\right)$ are independent random variables and each $I_{\alpha, \beta}\left(f_{j}\right)$ has the normal distribution with the mean 
$\int_{0}^{T} f_{j}(t) d \alpha(t)$ and the variance $\left\|f_{j}\right\|_{0, \beta}^{2}$. Moreover, if $f: \mathbb{R}^{n} \rightarrow$ $\mathbb{C}$ is Borel measurable, then

$$
\begin{gathered}
\int_{C[0, T]} f\left(I_{\alpha, \beta}\left(f_{1}\right)(x), \ldots, I_{\alpha, \beta}\left(f_{n}\right)(x)\right) d w_{\alpha, \beta ; \varphi}(x) \\
=\left[\prod_{j=1}^{n} \frac{1}{2 \pi\left\|f_{j}\right\|_{0, \beta}^{2}}\right]^{1 / 2} \int_{\mathbb{R}^{n}} f(\vec{u}) \exp \left\{-\frac{1}{2}\right. \\
\left.\cdot \sum_{j=1}^{n} \frac{\left[u_{j}-\int_{0}^{T} f_{j}(t) d \alpha(t)\right]^{2}}{\left\|f_{j}\right\|_{0, \beta}^{2}}\right\} d m_{L}^{n}(\vec{u}),
\end{gathered}
$$

where $\stackrel{*}{=}$ means that if either side exists, then both sides exist and they are equal.

Let $F: C[0, T] \rightarrow \mathbb{C}$ be a measurable function and suppose that the integral

$$
J_{F}(\lambda) \equiv \int_{C[0, T]} F\left(\lambda^{-1 / 2} x\right) d w_{\alpha, \beta ; \varphi}(x)
$$

exists as a finite number for all $\lambda>0$. If there exists a function $J_{F}^{*}(\lambda)$ analytic in $\mathbb{C}_{+} \equiv\{\lambda \in \mathbb{C}: \operatorname{Re} \lambda>0\}$ such that $J_{F}^{*}(\lambda)=J_{F}(\lambda)$ for all $\lambda>0$, then $J_{F}^{*}(\lambda)$ is defined to be a generalized analytic Wiener $w_{\alpha, \beta ; \varphi}$-integral of $F$ over $C[0, T]$ with parameter $\lambda$ and it is denoted by

$$
E_{w_{\alpha, \beta ;}}^{a n w_{\lambda}}[F]=J_{F}^{*}(\lambda)
$$

for $\lambda \in \mathbb{C}_{+}$. Let $q$ be a nonzero real number. If $E_{w_{\alpha, \beta ;}}^{a n w_{\lambda}}[F]$ has a limit as $\lambda$ approaches $-i q$ through $\mathbb{C}_{+}$, then we call it a generalized analytic Feynman $w_{\alpha, \beta ; \varphi}$-integral of $F$ over $C[0, T]$ with parameter $q$ and it is denoted by

$$
E_{w_{\alpha, \beta ; \varphi}}^{a n f_{q}}[F]=\lim _{\lambda \rightarrow-i q} E_{w_{\alpha, \beta ;}}^{a n w_{\lambda}}[F] .
$$

\section{A Banach Algebra with Its Applications}

In this section, we introduce a Banach algebra which generalizes the Banach algebra $\mathcal{S}$ given by Cameron and Storvick [1]. To define it, we need the following lemmas.

Lemma 3. Let $\mathscr{H}_{0}$ be a separable, real Hilbert space with the inner product $\langle\cdot, \cdot\rangle_{\mathscr{H}_{0}}$. Let $\mathscr{A}$ be the $\sigma$-algebra generated by the class of sets of the form

$$
\left\{g \in \mathscr{H}_{0}:\langle g, f\rangle_{\mathscr{H}_{0}}<r\right\},
$$

where $f$ and $r$ range over all elements in $\mathscr{H}_{0}$ and over all real numbers, respectively. Then we have $\mathscr{A}=\mathscr{B}\left(\mathscr{H}_{0}\right)$, where $\mathscr{B}\left(\mathscr{H}_{0}\right)$ is the Borel $\sigma$-algebra of $\mathscr{H}_{0}$.

Proof. Since $\langle\cdot, f\rangle_{\mathscr{H}_{0}}$ is continuous for each $f \in \mathscr{H}_{0}, \mathscr{B}\left(\mathscr{H}_{0}\right)$ contains all sets of the form given by (13) so that we have $\mathscr{A} \subseteq$ $\mathscr{B}\left(\mathscr{H}_{0}\right)$. To prove $\mathscr{B}\left(\mathscr{H}_{0}\right) \subseteq \mathscr{A}$, it suffices to show that for each $r>0$ and $f_{0} \in \mathscr{H}_{0}$, the closed ball

$$
B_{r, f_{0}} \equiv\left\{f \in \mathscr{H}_{0}:\left\|f-f_{0}\right\|_{\mathscr{H}_{0}} \leq r\right\}
$$

belongs to $\mathscr{A}$ since $\mathscr{H}_{0}$ is separable. Let $D \equiv\left\{f_{n}: n \in \mathbb{N}\right\}$ be a dense subset of $\mathscr{H}_{0}$. For each $n \in \mathbb{N}$, take $g_{n} \in \mathscr{H}_{0}$ such that $\left\langle g_{n}, f_{n}\right\rangle_{\mathscr{H}_{0}}=\left\|f_{n}\right\|_{\mathscr{H}_{0}}$ and $\left\|g_{n}\right\|_{\mathscr{H}_{0}}=1$ which can be justified by Corollary 14.13 of [12] as an application of the Hahn-Banach theorem and the Riesz representation theorem. For each $n \in$ $\mathbb{N}$, let

$$
B_{n, r, f_{0}}=\left\{f \in \mathscr{H}_{0}:\left|\left\langle f-f_{0}, g_{n}\right\rangle_{\mathscr{H}_{0}}\right| \leq r\right\} .
$$

Then for all positive integer $n$, we have

$$
\begin{aligned}
& B_{n, r, f_{0}}=\left\{f \in \mathscr{H}_{0}:\left\langle f_{0}, g_{n}\right\rangle_{\mathscr{H}_{0}}-r \leq\left\langle f, g_{n}\right\rangle_{\mathscr{H}_{0}}\right. \\
& \left.\quad \leq\left\langle f_{0}, g_{n}\right\rangle_{\mathscr{H}_{0}}+r\right\}
\end{aligned}
$$

which belongs to $\mathscr{A}$. We will prove $B_{r, f_{0}}=\bigcap_{n=1}^{\infty} B_{n, r, f_{0}}$ so that $B_{r, f_{0}}$ also belongs to $\mathscr{A}$. Indeed, if $f \in B_{r, f_{0}}$, then we have for all $n \in \mathbb{N}$

$$
\left|\left\langle f-f_{0}, g_{n}\right\rangle_{\mathscr{H}_{0}}\right| \leq\left\|f-f_{0}\right\|_{\mathscr{H}_{0}} \leq r
$$

by the Schwarz inequality, so that $f \in \bigcap_{n=1}^{\infty} B_{n, r, f_{0}}$; that is, $B_{r, f_{0}} \subseteq \bigcap_{n=1}^{\infty} B_{n, r, f_{0}}$. Conversely, let $f \in \bigcap_{n=1}^{\infty} B_{n, r, f_{0}}$ and let $\epsilon>0$ arbitrary. Since $D$ is a dense subset of $\mathscr{H}_{0}$, we can take $f_{n_{e}} \in D$ with $\left\|f-f_{0}-f_{n_{e}}\right\|_{\mathscr{H}_{0}}<\epsilon / 2$. Then we have by the Schwarz inequality

$$
\begin{aligned}
\left\|f-f_{0}\right\|_{\mathscr{H}_{0}} \leq & \left\|f-f_{0}-f_{n_{\epsilon}}\right\|_{\mathscr{H}_{0}}+\left\langle f_{n_{\epsilon}}, g_{n_{\epsilon}}\right\rangle_{\mathscr{H}_{0}} \\
= & \left\|f-f_{0}-f_{n_{\epsilon}}\right\|_{\mathscr{C}_{0}} \\
& +\left\langle f_{n_{\epsilon}}-f+f_{0}, g_{n_{\epsilon}}\right\rangle_{\mathscr{H}_{0}} \\
& +\left\langle f-f_{0}, g_{n_{\epsilon}}\right\rangle_{\mathscr{H}_{0}} \\
\leq & 2\left\|f-f_{0}-f_{n_{\epsilon}}\right\|_{\mathscr{H}_{0}}+\left|\left\langle f-f_{0}, g_{n_{\epsilon}}\right\rangle_{\mathscr{H}_{0}}\right| \\
< & \epsilon+r
\end{aligned}
$$

since $f \in B_{n_{e}, r, f_{0}}$. Since $\epsilon$ is arbitrary, we have $\left\|f-f_{0}\right\|_{\mathscr{H}_{0}} \leq r$ so that $f \in B_{r, f_{0}}$; that is, $\bigcap_{n=1}^{\infty} B_{n, r, f_{0}} \subseteq B_{r, f_{0}}$. Now the proof is completed as desired.

Remark 4. (1) By the Riesz representation theorem and Lemma $3, \mathscr{B}\left(\mathscr{H}_{0}\right)$ is in fact the smallest $\sigma$-algebra satisfying that all bounded linear functionals on $\mathscr{H}_{0}$ are measurable.

(2) Applying the method used in the proof of [13, Theorem 4.2, p. 74], we can also prove Lemma 3.

Lemma 5. (1) We have $L_{0, \beta}^{2}[0, T]=L^{2}[0, T]$ as vector spaces, where $L^{2}[0, T]$ denotes the Lebesgue space. Moreover, the two norms $\|\cdot\|_{0, \beta}$ and $\|\cdot\|_{m_{L}}$ are equivalent so that $\mathscr{B}\left(L_{0, \beta}^{2}[0, T]\right)=$ $\mathscr{B}\left(L^{2}[0, T]\right)$

(2) $\mathscr{B}\left(L_{\alpha, \beta}^{2}[0, T]\right)=\mathscr{B}\left(L_{0, \beta}^{2}[0, T]\right)$ if and only if $L_{\alpha, \beta}^{2}[0$, $T]=L_{0, \beta}^{2}[0, T]$ as vector spaces. In this case, the two norms $\|\cdot\|_{\alpha, \beta}$ and $\|\cdot\|_{0, \beta}$ are equivalent. 
Proof. Since $\beta^{\prime}$ is bounded away from 0 and continuous so that it is bounded on $[0, T]$, the two norms $\|\cdot\|_{0, \beta}$ and $\|\cdot\|_{m_{L}}$ are equivalent which implies $L_{0, \beta}^{2}[0, T]=L^{2}[0, T]$. Moreover, the identity operator from $\left(L_{0, \beta}^{2}[0, T],\|\cdot\|_{0, \beta}\right)$ to $\left(L^{2}[0, T],\|\cdot\|_{m_{L}}\right)$ is bicontinuous so that the topologies induced by $\|\cdot\|_{0, \beta}$ and $\|\cdot\|_{m_{L}}$ are equal. We now have $\mathscr{B}\left(L_{0, \beta}^{2}[0, T]\right)=\mathscr{B}\left(L^{2}[0, T]\right)$ which proves (1). If $\mathscr{B}\left(L_{\alpha, \beta}^{2}[0, T]\right)=\mathscr{B}\left(L_{0, \beta}^{2}[0, T]\right)$, then clearly we have $L_{\alpha, \beta}^{2}[0, T]=L_{0, \beta}^{2}[0, T]$. Conversely, if $L_{\alpha, \beta}^{2}[0, T]=L_{0, \beta}^{2}[0, T]$, then by the open mapping theorem, the identity operator $I:\left(L_{\alpha, \beta}^{2}[0, T],\|\cdot\|_{\alpha, \beta}\right) \rightarrow\left(L_{0, \beta}^{2}[0\right.$, $\left.T],\|\cdot\|_{0, \beta}\right)$ is a bounded operator and an open map since $\|f\|_{0, \beta} \leq\|f\|_{\alpha, \beta}$ for all $f \in L_{\alpha, \beta}^{2}[0, T]$; that is, $I$ is bicontinuous. Now the two norms $\|\cdot\|_{\alpha, \beta}$ and $\|\cdot\|_{0, \beta}$ are equivalent, and the topologies induced by $\|\cdot\|_{\alpha, \beta}$ and $\|\cdot\|_{0, \beta}$ are equal so that $\mathscr{B}\left(L_{\alpha, \beta}^{2}[0, T]\right)=\mathscr{B}\left(L_{0, \beta}^{2}[0, T]\right)$ which completes the proof of (2).

Example 6. (1) It is not difficult to show that $\alpha$ is a constant function on $[0, T]$ if and only if $\|f\|_{\alpha, 0}=0$ for all $f \in S[0, T]$ if and only if $\langle f, g\rangle_{\alpha, 0}=0$ for all $f, g \in S[0, T]$. In this case, $\langle f, g\rangle_{\alpha, \beta}=\langle f, g\rangle_{0, \beta}$ for all $f, g \in L_{0, \beta}^{2}[0, T]$ so that $L_{\alpha, \beta}^{2}[0, T]=L_{0, \beta}^{2}[0, T]$ isometrically which implies $\mathscr{B}\left(L_{\alpha, \beta}^{2}[0\right.$, $T])=\mathscr{B}\left(L_{0, \beta}^{2}[0, T]\right)$ by Lemma 5. In particular, if $\beta(t)=t$ for all $t \in[0, T]$, then $L_{\alpha, \beta}^{2}[0, T]=L^{2}[0, T]$ isometrically.

(2) If for some constant $c \geq 0,|\alpha|(t)=c \beta(t)$ for all $t \in$ $[0, T]$, which is the condition suggested by Yoo et al. [14], then $\langle f, g\rangle_{\alpha, \beta}=\langle f, g\rangle_{0,(1+c) \beta}$ so that $L_{\alpha, \beta}^{2}[0, T]=L_{0,(1+c) \beta}^{2}[0, T]$ isometrically. Since $L_{0,(1+c) \beta}^{2}[0, T]=L_{0, \beta}^{2}[0, T]$ as vector spaces, we have $L_{\alpha, \beta}^{2}[0, T]=L_{0, \beta}^{2}[0, T]$ as vector spaces, but they need not be equal isometrically. In this case, $\|\cdot\|_{\alpha, \beta}$ and $\|\cdot\|_{0, \beta}$ are equivalent and $\mathscr{B}\left(L_{\alpha, \beta}^{2}[0, T]\right)=\mathscr{B}\left(L_{0, \beta}^{2}[0, T]\right)$ by Lemma 5.

(3) It is obvious that $L_{\alpha, \beta}^{2}[0, T] \subseteq L_{0, \beta}^{2}[0, T]$. Suppose that $|\alpha|^{\prime}$ is bounded on $[0, T]$. For $f \in L_{0, \beta}^{2}[0, T]$, we have for some $M>0$

$$
\begin{aligned}
& \int_{0}^{T}[f(t)]^{2} d \nu_{\alpha, \beta}(t) \\
& \quad=\int_{0}^{T}[f(t)]^{2} \frac{(|\alpha|+\beta)^{\prime}(t)}{\beta^{\prime}(t)} d v_{\beta}(t) \leq M\|f\|_{0, \beta}^{2} \\
& \quad<\infty
\end{aligned}
$$

since $\beta^{\prime}$ is bounded and bounded away from 0 . We now have $L_{0, \beta}^{2}[0, T] \subseteq L_{\alpha, \beta}^{2}[0, T]$ and hence $L_{\alpha, \beta}^{2}[0, T]=L_{0, \beta}^{2}[0, T]$ as vector spaces, but they need not be equal isometrically. In this case, $\|\cdot\|_{\alpha, \beta}$ and $\|\cdot\|_{0, \beta}$ are equivalent and $\mathscr{B}\left(L_{\alpha, \beta}^{2}[0, T]\right)=$ $\mathscr{B}\left(L_{0, \beta}^{2}[0, T]\right)$ by Lemma 5 .

Throughout the remainder of this paper, we assume $L_{\alpha, \beta}^{2}[0, T]=L_{0, \beta}^{2}[0, T]$ as sets, unless otherwise specified. In this case, we have $L_{\alpha, \beta}^{2}[0, T]=L_{0, \beta}^{2}[0, T]=L^{2}[0, T]$ as vector spaces and $\mathscr{B} \equiv \mathscr{B}\left(L_{\alpha, \beta}^{2}[0, T]\right)=\mathscr{B}\left(L_{0, \beta}^{2}[0, T]\right)=$
$\mathscr{B}\left(L^{2}[0, T]\right)$ by Lemma 5 , but they need not be equal isometrically as Hilbert spaces. We also note that $L_{\alpha, \beta}^{2}[0, T]$ is separable since $L_{0, \beta}^{2}[0, T]$ is separable [10] and the two norms $\|\cdot\|_{\alpha, \beta}$ and $\|\cdot\|_{0, \beta}$ are equivalent by Lemma 5 . Let $\mathscr{M} \equiv$ $\mathscr{M}\left(L_{\alpha, \beta}^{2}[0, T]\right)$ be the class of complex measures of finite variation on $L_{\alpha, \beta}^{2}[0, T]$ with $\mathscr{B}$ as its $\sigma$-algebra of measurable sets. If $\mu \in \mathscr{M}$, then we set $\|\mu\|=\operatorname{var} \mu$, the total variation of $\mu$ over $L_{\alpha, \beta}^{2}[0, T]$. We also note that $\mathscr{M}\left(L_{\alpha, \beta}^{2}[0, T]\right)=\mathscr{M}\left(L_{0, \beta}^{2}[0, T]\right)$ and they are Banach algebras under convolution and with the total variation norm, since $L_{\alpha, \beta}^{2}[0, T]$ and $L_{0, \beta}^{2}[0, T]$ are separable, real, infinite dimensional Hilbert spaces [15].

We now have the following lemma.

Lemma 7. For $\mu \in \mathscr{M}, I_{\alpha, \beta}(f)(x)$ is a $\mu \times w_{\alpha, \beta ; \varphi}$-measurable function on $L_{\alpha, \beta}^{2}[0, T] \times C[0, T]$. Moreover, for $w_{\alpha, \beta ; \varphi}$ a.e. $x \in$ $C[0, T], I_{\alpha, \beta}(f)(x)$ exists for $\mu$ a.e. $f \in L_{\alpha, \beta}^{2}[0, T]$.

Proof. Note that each term of the right-hand side of $(7)$ is $\mathscr{B} \times$ $\mathscr{B}(C[0, T])$-measurable so that it is a $\mu \times w_{\alpha, \beta ; \varphi}$-measurable function on $L_{\alpha, \beta}^{2}[0, T] \times C[0, T]$. Since $I_{\alpha, \beta}(f)(x)=$ $\lim _{n \rightarrow \infty} \int_{0}^{T} \phi_{n}(t) d x(t), I_{\alpha, \beta}(f)(x)$ is also measurable with respect to the measure $\mu \times w_{\alpha, \beta ; \varphi}$ on $L_{\alpha, \beta}^{2}[0, T] \times C[0, T]$. Moreover, we have that, for $w_{\alpha, \beta ; \varphi}$ a.e. $x \in C[0, T], I_{\alpha, \beta}(f)(x)$ exists for $\mu$ a.e. $f \in L_{\alpha, \beta}^{2}[0, T]$.

Let $\mathcal{S}_{\alpha, \beta ; \varphi}$ be the space of functions of the form

$$
F^{\mu}(x)=\int_{L_{\alpha, \beta}^{2}[0, T]} \exp \left\{i I_{\alpha, \beta}(f)(x)\right\} d \mu(f)
$$

for all $x \in C[0, T]$ for which the integral exists, where $\mu \in \mathscr{M}$. We note that $F^{\mu}$ is well-defined for $w_{\alpha, \beta ; \varphi}$ a.e. $x \in C[0, T]$ by Lemma 7 and it is an integrable function of $x$ on $C[0, T]$. Moreover, $\mathcal{S}_{\alpha, \beta ; \varphi}$ is a linear space over $\mathbb{C}$.

We have the following uniqueness theorem.

Theorem 8. For $\mu \in \mathscr{M}$ and $F^{\mu} \in \mathcal{S}_{\alpha, \beta ; \varphi}$, let $F^{\mu}$ and $\mu$ be related by (20). Then $F^{\mu}$ is uniquely determined by $\mu$; that is, there is a unique $\mu \in \mathscr{M}$ such that (20) holds for $w_{\alpha, \beta ; \varphi}$ a.e. $x \in$ $C[0, T]$ so that there is an one-to-one correspondence between $\mathcal{S}_{\alpha, \beta ; \varphi}$ and $\mathscr{M}$.

Proof. Suppose that there are two measures $\mu_{1}$ and $\mu_{2}$ in $\mathscr{M}$ such that (20) holds with $F^{\mu_{1}}(x)=F^{\mu_{2}}(x)$ for $w_{\alpha, \beta ; \varphi}$ a.e. $x \in$ $C[0, T]$. Let $\mu_{3}=\mu_{1}-\mu_{2} \in \mathscr{M}$. Then for $w_{\alpha, \beta ; \varphi}$ a.e. $x \in C[0, T]$, we have

$$
\int_{L_{\alpha, \beta}^{2}[0, T]} \exp \left\{i I_{\alpha, \beta}(g)(x)\right\} d \mu_{3}(g)=0 .
$$

Let $r_{1}$ be any real number and let $f \in L_{\alpha, \beta}^{2}[0, T]$ with $\|f\|_{0, \beta} \neq$ 0 . Let $r=r_{1} /\|f\|_{0, \beta}^{2}$. If $-\infty \leq a<r$, then let $g_{a, r}(s)=\chi_{[a, r]}(s)$, 
and if $0<\delta<(r-a) / 2$, then let $g_{a, r, \delta}$ be the trapezoidal approximation

$$
g_{a, r, \delta}(s)= \begin{cases}\frac{s-a}{\delta} & \text { for } a \leq s<a+\delta \\ 1 & \text { for } a+\delta \leq s<r-\delta \\ \frac{r-s}{\delta} & \text { for } r-\delta \leq s \leq r \\ 0 & \text { elsewhere, }\end{cases}
$$

and let $g_{a, r, \delta}^{*}(s)=g_{a, r, \delta}(s) \exp \left\{-(1 / 2)\|f\|_{0, \beta}^{2} s^{2}\right\}$ for $s \in \mathbb{R}$. Then $g_{a, r, \delta}^{*}(s)$ is a bounded continuous function of class $L^{1}(\mathbb{R})$ having a piecewise continuous derivative of bounded variation and vanishing outside a finite interval. Consequently, its Fourier transform $\mathscr{F}\left(g_{a, r, \delta}^{*}\right)$ is a bounded continuous function of class $L^{1}(\mathbb{R})$ and we have

$$
\begin{aligned}
& \mathscr{F}\left(g_{a, r, \delta}^{*}\right)(u)=\left(\frac{1}{2 \pi}\right)^{1 / 2} \\
& \cdot \int_{\mathbb{R}} \exp \{-i s u\} g_{a, r, \delta}^{*}(s) d m_{L}(s) \quad \text { for } u \in \mathbb{R}, \\
& g_{a, r, \delta}^{*}(s)=\left(\frac{1}{2 \pi}\right)^{1 / 2} \\
& \quad \cdot \int_{\mathbb{R}} \exp \{i s u\} \mathscr{F}\left(g_{a, r, \delta}^{*}\right)(u) d m_{L}(u) \quad \text { for } s \in \mathbb{R} .
\end{aligned}
$$

For $g \in L_{\alpha, \beta}^{2}[0, T]$, let

$$
\theta(t)=g(t)-\frac{\langle g, f\rangle_{0, \beta}}{\|f\|_{0, \beta}^{2}} f(t)
$$

for $v_{\alpha, \beta}$ a.e. $t \in[0, T]$. Then (24) holds for $v_{\beta}$ a.e. $t \in[0, T]$ which implies $\langle\theta, f\rangle_{0, \beta}=\langle g, f\rangle_{0, \beta}-\langle g, f\rangle_{0, \beta}=0$ and

$$
\|\theta\|_{0, \beta}^{2}=\|g\|_{0, \beta}^{2}-\frac{\langle g, f\rangle_{0, \beta}^{2}}{\|f\|_{0, \beta}^{2}} .
$$

Suppose that $\theta \neq 0$ in $L_{\alpha, \beta}^{2}[0, T]$. Then $\theta \neq 0$ in $L_{0, \beta}^{2}[0, T]$. By Theorem 2, it is not difficult to show that

$$
\begin{aligned}
& G_{f}(x) \equiv \mathscr{F}\left(g_{a, r, \delta}^{*}\right)\left(I_{\alpha, \beta}(f)(x)\right) \\
& \cdot \exp \left\{\frac{1}{2\|f\|_{0, \beta}^{2}}\left[I_{\alpha, \beta}(f)(x)-\int_{0}^{T} f(t) d \alpha(t)\right]^{2}\right\}
\end{aligned}
$$

is an integrable function of $x$ with respect to $w_{\alpha, \beta ; \varphi}$ over $C[0, T]$. Now we have by Theorem 2 and the linearity of $I_{\alpha, \beta}$

$$
\begin{aligned}
& \int_{C[0, T]} G_{f}(x) \exp \left\{i I_{\alpha, \beta}(g)(x)\right\} d w_{\alpha, \beta ; \varphi}(x) \\
& \quad=\int_{C[0, T]} \mathscr{F}\left(g_{a, r, \delta}^{*}\right)\left(I_{\alpha, \beta}(f)(x)\right) \\
& \quad \cdot \exp \left\{\frac{1}{2\|f\|_{0, \beta}^{2}}\left[I_{\alpha, \beta}(f)(x)-\int_{0}^{T} f(t) d \alpha(t)\right]^{2}\right\}
\end{aligned}
$$

$$
\begin{aligned}
& \times \exp \left\{i\left[I_{\alpha, \beta}(\theta)(x)+\frac{\langle g, f\rangle_{0, \beta}}{\|f\|_{0, \beta}^{2}} I_{\alpha, \beta}(f)(x)\right]\right\} d w_{\alpha, \beta ; \varphi}(x) \\
& =\frac{1}{2 \pi\|f\|_{0, \beta}\|\theta\|_{0, \beta}} \int_{\mathbb{R}^{2}} \mathscr{F}\left(g_{a, r, \delta}^{*}\right)\left(u_{1}\right) \\
& \cdot \exp \left\{-\frac{1}{2\|\theta\|_{0, \beta}^{2}}\left[u_{2}-\int_{0}^{T} \theta(t) d \alpha(t)\right]^{2}\right. \\
& \left.+i\left[u_{2}+\frac{\langle g, f\rangle_{0, \beta}}{\|f\|_{0, \beta}^{2}} u_{1}\right]\right\} d m_{L}^{2}\left(u_{1}, u_{2}\right) \\
& =\left(\frac{1}{2 \pi\|f\|_{0, \beta}^{2}}\right)^{1 / 2} \int_{\mathbb{R}} \mathscr{F}\left(g_{a, r, \delta}^{*}\right)\left(u_{1}\right) \\
& \cdot \exp \left\{-\frac{1}{2}\left[\|g\|_{0, \beta}^{2}-\frac{\langle g, f\rangle_{0, \beta}^{2}}{\|f\|_{0, \beta}^{2}}\right]\right. \\
& \left.+i\left[\int_{0}^{T} \theta(t) d \alpha(t)+\frac{\langle g, f\rangle_{0, \beta}}{\|f\|_{0, \beta}^{2}} u_{1}\right]\right\} d m_{L}\left(u_{1}\right) \\
& =\frac{1}{\|f\|_{0, \beta}} g_{a, r, \delta}^{*}\left(\frac{\langle g, f\rangle_{0, \beta}}{\|f\|_{0, \beta}^{2}}\right)_{\exp }\left\{-\frac{1}{2}\left[\|g\|_{0, \beta}^{2}-\frac{\langle g, f\rangle_{0, \beta}^{2}}{\|f\|_{0, \beta}^{2}}\right]\right. \\
& \left.+i \int_{0}^{T} \theta(t) d \alpha(t)\right\}=\frac{1}{\|f\|_{0, \beta}} g_{a, r, \delta}\left(\frac{\langle g, f\rangle_{0, \beta}}{\|f\|_{0, \beta}^{2}}\right) \\
& \cdot \exp \left\{-\frac{1}{2}\|g\|_{0, \beta}^{2}+i \int_{0}^{T} \theta(t) d \alpha(t)\right\}
\end{aligned}
$$

which still holds for $\theta=0$ in $L_{\alpha, \beta}^{2}[0, T]$ since $\theta=0$ in $L_{\alpha, \beta}^{2}[0, T]$ implies $\theta=0$ in both $L_{\alpha, 0}^{2}[0, T]$ and $L_{0, \beta}^{2}[0, T]$. We now have by the Fubini theorem

$$
\begin{aligned}
0= & \int_{L_{\alpha, \beta}^{2}[0, T]} \int_{C[0, T]} G_{f}(x) \\
& \cdot \exp \left\{i I_{\alpha, \beta}(g)(x)\right\} d w_{\alpha, \beta ; \varphi}(x) d \mu_{3}(g) \\
& =\frac{1}{\|f\|_{0, \beta}} \int_{L_{\alpha, \beta}^{2}[0, T]} g_{a, r, \delta}\left(\frac{\langle g, f\rangle_{0, \beta}}{\|f\|_{0, \beta}^{2}}\right) d \mu_{f}(g),
\end{aligned}
$$

where $\mu_{f}$ is the measure on $\mathscr{B}$ defined by

$$
\frac{d \mu_{f}}{d \mu_{3}}(g)=\exp \left\{-\frac{1}{2}\|g\|_{0, \beta}^{2}+i \int_{0}^{T} \theta(t) d \alpha(t)\right\}
$$

for $\mu_{3}$ a.e. $g \in L_{\alpha, \beta}^{2}[0, T]$. Note that $\left|g_{a, r, \delta}\left(\langle g, f\rangle_{0, \beta} /\|f\|_{0, \beta}^{2}\right)\right| \leq$ 1 for all $g$ and $\delta$. Letting $\delta \rightarrow 0^{+}$, we have by the dominated convergence theorem

$$
0=\frac{1}{\|f\|_{0, \beta}} \int_{L_{\alpha, \beta}^{2}[0, T]} g_{a, r}\left(\frac{\langle g, f\rangle_{0, \beta}}{\|f\|_{0, \beta}^{2}}\right) d \mu_{f}(g) .
$$


Also, letting $a \rightarrow-\infty$, we have by the dominated convergence theorem again

$$
0=\frac{1}{\|f\|_{0, \beta}} \int_{L_{\alpha, \beta}^{2}[0, T]} g_{-\infty, r}\left(\frac{\langle g, f\rangle_{0, \beta}}{\|f\|_{0, \beta}^{2}}\right) d \mu_{f}(g)
$$

so that if

$$
E_{r_{1}}=\left\{g \in L_{\alpha, \beta}^{2}[0, T]:\langle g, f\rangle_{0, \beta} \leq r_{1}\right\},
$$

then we have $\mu_{f}\left(E_{r_{1}}\right)=0$. If $\|f\|_{0, \beta}=0$, then $E_{r_{1}}$ is either $\emptyset$ or $L_{\alpha, \beta}^{2}[0, T]$. Let $\mu_{0}$ be the measure defined by (29) with replacing $f$ by 1 . If $E_{r_{1}}=\emptyset$, then $\mu_{0}\left(E_{r_{1}}\right)=0$. If $E_{r_{1}}=L_{\alpha, \beta}^{2}[0$, $T]$, then

$$
\begin{aligned}
\mu_{0}\left(E_{r_{1}}\right) & =\lim _{n \rightarrow \infty} \mu_{0}\left(\left\{g \in L_{\alpha, \beta}^{2}[0, T]:\langle g, 1\rangle_{0, \beta} \leq n\right\}\right) \\
& =0
\end{aligned}
$$

by the above argument since $\|1\|_{0, \beta}>0$. Solving (29) for $\mu_{3}$, we have for all $f \in L_{\alpha, \beta}^{2}[0, T]$

$$
\begin{aligned}
\mu_{3} & \left(E_{r_{1}}\right) \\
& =\int_{E_{r_{1}}} \exp \left\{\frac{1}{2}\|g\|_{0, \beta}^{2}-i \int_{0}^{T} \theta(t) d \alpha(t)\right\} d \mu_{f}(g) \\
& =0 .
\end{aligned}
$$

Since $L_{\alpha, \beta}^{2}[0, T]=L_{0, \beta}^{2}[0, T], \mathscr{B}$ is generated by the sets of the form $E_{r_{1}}$ so that we have $\mu_{1}(E)-\mu_{2}(E)=\mu_{3}(E)=0$ for all $E \in \mathscr{B}$, that is, $\mu_{1}=\mu_{2}$, which completes the proof.

Remark 9. A difference between the proof of Theorem 8 and the proof of Theorem 2.1 in [1] is to use the additional condition $L_{\alpha, \beta}^{2}[0, T]=L_{0, \beta}^{2}[0, T]$ as sets in Theorem 8. In order to apply Theorem 2 to the proof of Theorem 8 , we need an orthonormalization process in the Hilbert space $L_{0, \beta}^{2}[0, T]$ for the functions in the Hilbert space $L_{\alpha, \beta}^{2}[0, T]$. See (2) of Lemma 5.

Example 10. For some constant $c \geq 0$, let $|\alpha|(t)=c \beta(t)$ for all $t \in[0, T]$. Then we have $L_{\alpha, \beta}^{2}[0, T]=L_{0,(1+c) \beta}^{2}[0, T]$ isometrically. Replacing $\alpha$ and $\beta$ by the zero function and $(1+c) \beta$, respectively, in the proof of Theorem 8 , we can show that $F^{\mu}$ is uniquely determined by $\mu \in \mathscr{M}$.

Corollary 11. Suppose that $\alpha$ is a constant function on $[0, T]$. Let $F(\cdot, y) \in \mathcal{S}_{\alpha, \beta ; \varphi}$ for each $y \in Y$, where $Y$ is a measure space, and let $F(x, y)$ be a measurable function of $(x, y)$ on $C[0, T] \times$ $Y$. Let $\left\{\mu_{y}\right\}$ be the family of measures corresponding to $F(\cdot, y)$ so that for each $y \in Y$ and $\mu_{y} \in \mathscr{M}$,

$$
F(x, y)=\int_{L_{\alpha, \beta}^{2}[0, T]} \exp \left\{i I_{\alpha, \beta}(f)(x)\right\} d \mu_{y}(f)
$$

for $w_{\alpha, \beta ; \varphi}$ a.e. $x \in C[0, T]$. Then, for each $E \in \mathscr{B}, \mu_{y}(E)$ is measurable as a function of $y$ on $Y$.
Proof. This corollary follows from the fact that the method of proof of Theorem 8 has provided a method for explicitly constructing $\mu_{y}$ in terms of $F(\cdot, y)$. We will use the same assumptions and notations in the proof of Theorem 8. Indeed, for $f \in L_{\alpha, \beta}^{2}[0, T]$ with $\|f\|_{0, \beta} \neq 0$, let

$$
K_{a, r, \delta}^{f}(y)=\int_{C[0, T]} G_{f}(x) F(x, y) d w_{\alpha, \beta ; \varphi}(x) .
$$

It is a measurable function of $y$ by the assumption. Then we have

$$
\begin{aligned}
& K_{a, r, \delta}^{f}(y)=\int_{L_{\alpha, \beta}^{2}[0, T]} \int_{C[0, T]} G_{f}(x) \\
& \quad \cdot \exp \left\{i I_{\alpha, \beta}(g)(x)\right\} d w_{\alpha, \beta ; \varphi}(x) d \mu_{y}(g) \\
& =\frac{1}{\|f\|_{0, \beta}} \int_{L_{\alpha, \beta}^{2}[0, T]} g_{a, r, \delta}\left(\frac{\langle g, f\rangle_{0, \beta}}{\|f\|_{0, \beta}^{2}}\right) d \zeta_{y}(g),
\end{aligned}
$$

where $\left(d \zeta_{y} / d \mu_{y}\right)(g)=\exp \left\{-(1 / 2)\|g\|_{0, \beta}^{2}\right\}$ for $\mu_{y}$ a.e. $g \in$ $L_{\alpha, \beta}^{2}[0, T]$. Letting $\delta \rightarrow 0^{+}$, we have by the dominated convergence theorem

$$
\begin{aligned}
& \lim _{\delta \rightarrow 0^{+}} K_{a, r, \delta}^{f}(y) \\
& =\frac{1}{\|f\|_{0, \beta}} \int_{L_{\alpha, \beta}^{2}[0, T]} g_{a, r}\left(\frac{\langle g, f\rangle_{0, \beta}}{\|f\|_{0, \beta}^{2}}\right) d \zeta_{y}(g)
\end{aligned}
$$

which is still a measurable function of $y$. Letting $a \rightarrow-\infty$, we have by the dominated convergence theorem again

$$
\begin{aligned}
& \lim _{a \rightarrow-\infty} \lim _{\delta \rightarrow 0^{+}} K_{a, r, \delta}^{f}(y) \\
& =\frac{1}{\|f\|_{0, \beta}} \int_{L_{\alpha, \beta}^{2}[0, T]} g_{-\infty, r}\left(\frac{\langle g, f\rangle_{0, \beta}}{\|f\|_{0, \beta}^{2}}\right) d \zeta_{y}(g)
\end{aligned}
$$

so that if

$$
E_{r_{1}}=\left\{g \in L_{\alpha, \beta}^{2}[0, T]:\langle g, f\rangle_{0, \beta} \leq r_{1}\right\},
$$

then $\zeta_{y}\left(E_{r_{1}}\right)$ is a measurable function of $y$ on $Y$. If $\|f\|_{0, \beta}=0$, $E_{r_{1}}$ is either $\emptyset$ or $L_{\alpha, \beta}^{2}[0, T]$. If $E_{r_{1}}=\emptyset$, then $0=\zeta_{y}\left(E_{r_{1}}\right)$ which is a measurable function of $y$. If $E_{r_{1}}=L_{\alpha, \beta}^{2}[0, T]$, then we have

$$
\zeta_{y}\left(E_{r_{1}}\right)=\lim _{n \rightarrow \infty} \zeta_{y}\left(\left\{g \in L_{\alpha, \beta}^{2}[0, T]:\langle g, 1\rangle_{0, \beta} \leq n\right\}\right)
$$

which is also a measurable function of $y$ by the above argument. Since $\mathscr{B}$ is generated by the sets of the form $E_{r_{1}}$, $\zeta_{y}(E)$ is a measurable function of $y$ on $Y$ for all $E \in \mathscr{B}$. Solving $\mu_{y}$ for $\zeta_{y}$, we have for all $E \in \mathscr{B}$

$$
\mu_{y}(E)=\int_{E} \exp \left\{\frac{1}{2}\|g\|_{0, \beta}^{2}\right\} d \zeta_{y}(g)
$$

which is a measurable function of $y$ on $Y$, since the integrand can be expressed by a limit of simple functions on $L_{\alpha, \beta}^{2}[0, T]$. 
Definition 12. If $\mu \in \mathscr{M}$ and $F^{\mu} \in \mathcal{S}_{\alpha, \beta ; \varphi}$ are related by (20), we define a norm $\left\|F^{\mu}\right\|$ of $F^{\mu}$ by $\left\|F^{\mu}\right\|=\|\mu\|$. We note that $\left\|F^{\mu}\right\|$ is well defined by Theorem 8 .

Using the same process used in the proof of Theorem 2.3 in [1], we can prove the following theorem.

Theorem 13. $\mathcal{S}_{\alpha, \beta ; \varphi}$ is a normed algebra. Moreover, the correspondence $\mu \rightarrow F^{\mu}$ given by (20) for $\mu \in \mathscr{M}$ is an algebra isometric isomorphism between $\mathscr{M}$ and $\mathcal{S}_{\alpha, \beta ; \varphi}$, so that $\mathcal{S}_{\alpha, \beta ; \varphi}$ is a Banach algebra.

Remark 14. (1) One can show that $\mathcal{S}_{\alpha, \beta ; \varphi}$ is a Banach space without the isomorphism in Theorem 13. For more details, see the proof of Theorem 2.2 in [1].

(2) If $\alpha(t)=0$ and $\beta(t)=t$ for all $t \in[0, T]$, and $\varphi$ is the Dirac measure concentrated at 0 , then we can obtain all results of Section 2 in [1] from Theorems 8 and 13 so that $\mathcal{S}_{\alpha, \beta ; \varphi}$ generalizes the Banach algebra $\mathcal{S}$ introduced by Cameron and Storvick [1].

\section{The Fresnel Class with Its Equivalent Spaces}

In this section, we establish that the Fresnel class $[4,5]$ is isometrically isomorphic to $\mathcal{S}_{\alpha, \beta ; \varphi}$. Some ideas of the results in this section follow from [4,7], but the detailed proofs of the results in this section are quite different from those in $[4,7]$.

Let $\mathscr{H}$ be the space of real-valued functions $v$ on $[0, T]$ which are absolutely continuous with $v(0)=0$ and $v^{\prime} / \beta^{\prime} \in$ $L_{\alpha, \beta}^{2}[0, T]$. Define $D: \mathscr{H} \rightarrow L_{\alpha, \beta}^{2}[0, T]$ by $D v=v^{\prime} / \beta^{\prime}$ for $v \in \mathscr{H}$, and an inner product $\langle\cdot, \cdot\rangle_{\mathscr{H}}$ on $\mathscr{H}$ by

$$
\left\langle v_{1}, v_{2}\right\rangle_{\mathscr{H}}=\left\langle D v_{1}, D v_{2}\right\rangle_{0, \beta}=\int_{0}^{T} \frac{v_{1}^{\prime}(t) v_{2}^{\prime}(t)}{\beta^{\prime}(t)} d m_{L}(t)
$$

for $v_{1}, v_{2} \in \mathscr{H}$, which can be justified by the fact $L_{\alpha, \beta}^{2}[0, T]=$ $L_{0, \beta}^{2}[0, T]$ as vector spaces.

We now have the following results.

Lemma 15. $\langle\cdot, \cdot\rangle_{\mathscr{H}}$ is well defined on $\mathscr{H} \times \mathscr{H}$ and $\left(\mathscr{H},\langle\cdot, \cdot\rangle_{\mathscr{H}}\right)$ is a real inner product space.

Proof. By the Minkowski inequality, it is clear that $\mathscr{H}$ is a real linear space. Let $v_{1}, v_{2} \in \mathscr{H}$. If $D v_{1}(t)=z_{1}(t)$ and $D v_{2}(t)=$ $z_{2}(t)$ for $v_{\alpha, \beta}$ a.e. $t \in[0, T]$, then $D v_{1}(t)=z_{1}(t)$ and $D v_{2}(t)=$ $z_{2}(t)$ for $v_{\beta}$ a.e. $t \in[0, T]$ so that we have

$$
\left\langle v_{1}, v_{2}\right\rangle_{\mathscr{C}}=\left\langle D v_{1}, D v_{2}\right\rangle_{0, \beta}=\left\langle z_{1}, z_{2}\right\rangle_{0, \beta},
$$

that is, $\langle\cdot, \cdot\rangle_{\mathscr{H}}$ is well defined. It is obvious that $\langle\cdot, \cdot\rangle_{\mathscr{H}}$ is a symmetric, bilinear, nonnegative definite form on $\mathscr{H} \times \mathscr{H}$. Moreover, if $\langle v, v\rangle_{\mathscr{H}}=0$ for $v \in \mathscr{H}$, then $\left[v^{\prime}(t)\right]^{2} / \beta^{\prime}(t)=0$ for $m_{L}$ a.e. $t \in[0, T]$ so that $v^{\prime}(t)=0$ for $m_{L}$ a.e. $t \in[0, T]$ since $\beta^{\prime}$ is bounded away from 0 . Now we have for all $t \in[0, T]$

$$
v(t)=\int_{0}^{t} v^{\prime}(s) d m_{L}(s)+v(0)=0
$$

so that $\langle\cdot, \cdot\rangle_{\mathscr{H}}$ is positive definite. Hence, the theorem is proved.
Lemma 16. Define $I: L_{\alpha, \beta}^{2}[0, T]\left(=L_{0, \beta}^{2}[0, T]\right) \rightarrow \mathscr{H}$ by

$$
\text { (If) }(t)=\int_{0}^{t} f(s) \beta^{\prime}(s) d m_{L}(s)
$$

for $f \in L_{\alpha, \beta}^{2}[0, T]$ and for $t \in[0, T]$. Then $D: \mathscr{H} \rightarrow L_{\alpha, \beta}^{2}[0$, $T]\left(=L_{0, \beta}^{2}[0, T]\right)$ is a vector space isomorphism and $I=D^{-1}$. Moreover, $D$ and $I$ are isometric isomorphisms between $\mathscr{H}$ and $L_{0, \beta}^{2}[0, T]$ so that $\mathscr{H}$ is a separable, infinite dimensional Hilbert space, and both $D: \mathscr{H} \rightarrow L_{\alpha, \beta}^{2}[0, T]$ and $I: L_{\alpha, \beta}^{2}[0, T] \rightarrow \mathscr{H}$ are bounded linear operators.

Proof. Clearly, $D$ and $I$ are well defined and linear. Moreover, $D I f=f$ for all $f \in L_{\alpha, \beta}^{2}[0, T]$ and $(I D v)(t)=v(t)-v(0)=$ $v(t)$ for all $t \in[0, T]$ if $v \in \mathscr{H}$. These equalities tell us that $I D v=v$ for all $v \in \mathscr{H}$, which implies that $D$ is a vector space isomorphism and $I=D^{-1}$. For $v_{1}, v_{2} \in \mathscr{H}$, we have $\left\langle v_{1}, v_{2}\right\rangle_{\mathscr{C}}=\left\langle D v_{1}, D v_{2}\right\rangle_{0, \beta}$ by the definition of $\langle\cdot, \cdot\rangle_{\mathscr{H}}$ so that $D$ and $I$ are isometric isomorphisms between $\mathscr{H}$ and $L_{0, \beta}^{2}[0, T]$. Now, $\mathscr{H}$ is a separable, infinite dimensional Hilbert space since $L_{0, \beta}^{2}[0, T]$ is. Moreover, for $f \in L_{\alpha, \beta}^{2}[0, T]$, we have

$$
\|I f\|_{\mathscr{H}}=\|D I f\|_{0, \beta}=\|f\|_{0, \beta} \leq\|f\|_{\alpha, \beta}
$$

so that $I: L_{\alpha, \beta}^{2}[0, T] \rightarrow \mathscr{H}$ is bounded. Since $I$ is onto, $I=$ $D^{-1}$ is an open map by the open mapping theorem so that $D$ is bounded.

Remark 17. (1) We have $L_{\alpha, \beta}^{2}[0, T]=L_{0, \beta}^{2}[0, T]$ as vector spaces, but we note that it need not hold isometrically as Hilbert spaces since the inner products $\langle\cdot, \cdot\rangle_{\alpha, \beta}$ and $\langle\cdot, \cdot\rangle_{0, \beta}$ need not be equal. Thus, $D$ and $I$ need not be isometric isomorphisms between $\mathscr{H}$ and $L_{\alpha, \beta}^{2}[0, T]$ in Lemma 16 .

(2) Both $D: \mathscr{H} \rightarrow L_{\alpha, \beta}^{2}[0, T]$ and $I: L_{\alpha, \beta}^{2}[0, T] \rightarrow \mathscr{H}$ are bounded linear operators by Lemma 16 so that $D: \mathscr{H} \rightarrow$ $L_{\alpha, \beta}^{2}[0, T]$ and $I: L_{\alpha, \beta}^{2}[0, T] \rightarrow \mathscr{H}$ are $\mathscr{B}(\mathscr{H})-\mathscr{B}$ and $\mathscr{B}$ $\mathscr{B}(\mathscr{H})$ measurable, respectively, where $\mathscr{B}(\mathscr{H})$ denotes the Borel $\sigma$-algebra of $\mathscr{H}$.

Let $\mathscr{M}(\mathscr{H})$ be the collection of $\mathbb{C}$-valued, countably additive measures of finite variation on $\mathscr{B}(\mathscr{H})$. Define $\mathscr{D}$ : $\mathscr{M}(\mathscr{H}) \rightarrow \mathscr{M}$ by $\mathscr{D} \sigma=\sigma \circ D^{-1}$ for $\sigma \in \mathscr{M}(\mathscr{H})$ and $\mathscr{I}: \mathscr{M} \rightarrow \mathscr{M}(\mathscr{H})$ by $\mathscr{I} \mu=\mu \circ I^{-1}$ for $\mu \in \mathscr{M}$. We note that $\mathscr{M}=\mathscr{M}\left(L_{\alpha, \beta}^{2}[0, T]\right)=\mathscr{M}\left(L_{0, \beta}^{2}[0, T]\right)$ with $\mathscr{B}$ as its $\sigma$-algebra of measurable sets and both $\mathscr{D}$ and $\mathscr{I}$ are well defined by the arguments in (2) of Remark 17. Moreover, $\mathscr{M}(\mathscr{H})$ is a Banach algebra under convolution and with the total variation norm, since $\mathscr{H}$ is a separable, infinite dimensional, real Hilbert space by Lemmas 15 and 16 [15].

Lemma 18. $\mathscr{D}$ is a Banach algebra isometric isomorphism between $\mathscr{M}(\mathscr{H})$ and $\mathscr{M}$ with $\mathscr{D}^{-1}=\mathscr{I}$.

Proof. Clearly, $\mathscr{D}$ is linear and bijective with $\mathscr{D}^{-1}=\mathscr{I}$. To prove that $\mathscr{D}$ preserves convolutions, let $\sigma_{1}, \sigma_{2} \in \mathscr{M}(\mathscr{H})$. For $B \in \mathscr{B}$, we have

$$
\begin{aligned}
& {\left[\left(\mathscr{D} \sigma_{1}\right) *\left(\mathscr{D} \sigma_{2}\right)\right](B)} \\
& =\int_{L_{\alpha, \beta}^{2}[0, T]} \int_{L_{\alpha, \beta}^{2}[0, T]} \chi_{B}(f+g) d \mathscr{D} \sigma_{1}(f) d \mathscr{D} \sigma_{2}(g) .
\end{aligned}
$$


For each $g \in L_{\alpha, \beta}^{2}[0, T]$, define $T_{g}: L_{\alpha, \beta}^{2}[0, T] \rightarrow L_{\alpha, \beta}^{2}[0, T]$ by $T_{g}(f)=f+g$ for $f \in L_{\alpha, \beta}^{2}[0, T]$. Then $T_{g}$ is continuous so that it is $\mathscr{B}-\mathscr{B}$ measurable and $T_{g}^{-1}(B)=B-g \in \mathscr{B}$. Now we have by the change of variable theorem

$$
\begin{aligned}
& {\left[\left(\mathscr{D} \sigma_{1}\right) *\left(\mathscr{D} \sigma_{2}\right)\right](B)} \\
& =\int_{L_{\alpha, \beta}^{2}[0, T]} \int_{L_{\alpha, \beta}^{2}[0, T]} \chi_{B}(f) d\left(\mathscr{D} \sigma_{1} \circ T_{g}^{-1}\right)(f) d \mathscr{D} \sigma_{2}(g) \\
& =\int_{L_{\alpha, \beta}^{2}[0, T]}\left(\mathscr{D} \sigma_{1} \circ T_{g}^{-1}\right)(B) d \mathscr{D} \sigma_{2}(g) \\
& =\int_{L_{\alpha, \beta}^{2}[0, T]} \sigma_{1}\left[D^{-1}(B-g)\right] d\left(\sigma_{2} \circ D^{-1}\right)(g) \\
& =\int_{\mathscr{H}} \sigma_{1}\left[D^{-1}(B)-u\right] d \sigma_{2}(u) .
\end{aligned}
$$

For each $u \in \mathscr{H}$, define $S_{u}: \mathscr{H} \rightarrow \mathscr{H}$ by $S_{u}(v)=v+u$ for $v \in \mathscr{H}$. By the same argument as above, we have

$$
\begin{aligned}
& {\left[\left(\mathscr{D} \sigma_{1}\right) *\left(\mathscr{D} \sigma_{2}\right)\right](B)} \\
& \quad=\int_{\mathscr{H}} \sigma_{1}\left[S_{u}^{-1}\left[D^{-1}(B)\right]\right] d \sigma_{2}(u) \\
& \quad=\int_{\mathscr{H}} \int_{\mathscr{H}} \chi_{D^{-1}(B)}(v) d\left(\sigma_{1} \circ S_{u}^{-1}\right)(v) d \sigma_{2}(u) \\
& \quad=\int_{\mathscr{C}} \int_{\mathscr{C}} \chi_{D^{-1}(B)}(v+u) d \sigma_{1}(v) d \sigma_{2}(u) \\
& =\left(\sigma_{1} * \sigma_{2}\right)\left[D^{-1}(B)\right]=\left[\mathscr{D}\left(\sigma_{1} * \sigma_{2}\right)\right](B),
\end{aligned}
$$

that is, $\left(\mathscr{D} \sigma_{1}\right)^{*}\left(\mathscr{D} \sigma_{2}\right)=\mathscr{D}\left(\sigma_{1} * \sigma_{2}\right)$ so that $\mathscr{D}$ is an algebra isomorphism. To complete the proof, we have to show $\|\mathscr{D} \sigma\|=\|\sigma\|$ for $\sigma \in \mathscr{M}(\mathscr{H})$, where $\|\cdot\|$ denotes the total variation norm on each space. Indeed, we have by the Riesz representation theorem and the change of variable theorem

$$
\begin{aligned}
& \|\mathscr{D} \sigma\|=\sup \left\{\left|\int_{L_{\alpha, \beta}^{2}[0, T]} v(f) d(\mathscr{D} \sigma)(f)\right|: v\right. \\
& \left.\in C\left(L_{\alpha, \beta}^{2}[0, T]\right), \sup _{f \in L_{\alpha, \beta}^{2}[0, T]}|v(f)|=1\right\} \\
& =\sup \left\{\left|\int_{L_{\alpha, \beta}^{2}[0, T]} v(f) d\left(\sigma \circ D^{-1}\right)(f)\right|: v\right. \\
& \left.\in C\left(L_{\alpha, \beta}^{2}[0, T]\right), \sup _{f \in L_{\alpha, \beta}^{2}[0, T]}|v(f)|=1\right\} \\
& =\sup \left\{\left|\int_{\mathscr{H}}(v \circ D)(u) d \sigma(u)\right|: v\right. \\
& \left.\quad \in C\left(L_{\alpha, \beta}^{2}[0, T]\right), \sup _{f \in L_{\alpha, \beta}^{2}[0, T]}|v(f)|=1\right\},
\end{aligned}
$$

where $C\left(L_{\alpha, \beta}^{2}[0, T]\right)$ denotes the set of all $\mathbb{C}$-valued, bounded, and continuous functions on $L_{\alpha, \beta}^{2}[0, T]$. Let $C(\mathscr{H})$ denote the set of all $\mathbb{C}$-valued, bounded, and continuous functions on $\mathscr{H}$. We now have that if $v \in C\left(L_{\alpha, \beta}^{2}[0, T]\right)$, then $v \circ D \in C(\mathscr{H})$ and

$$
\begin{aligned}
\sup & \{|(v \circ D)(u)|: u \in \mathscr{H}\} \\
= & \sup \left\{|v(f)|: f \in L_{\alpha, \beta}^{2}[0, T]\right\}
\end{aligned}
$$

since $D$ is a continuous isomorphism from $\mathscr{H}$ onto $L_{\alpha, \beta}^{2}[0, T]$ by Lemma 16. Similarly, if $g \in C(\mathscr{H})$, then $g \circ I \in$ $C\left(L_{\alpha, \beta}^{2}[0, T]\right)$ and

$$
\begin{aligned}
\sup & \left\{|(g \circ I)(f)|: f \in L_{\alpha, \beta}^{2}[0, T]\right\} \\
= & \sup \{|g(u)|: u \in \mathscr{H}\}
\end{aligned}
$$

by Lemma 16. Thus, we have

$$
\begin{aligned}
& \|\mathscr{D} \sigma\|=\sup \left\{\left|\int_{\mathscr{H}} g(u) d \sigma(u)\right|: g\right. \\
& \left.\quad \in C(\mathscr{H}), \sup _{u \in \mathscr{H}}|g(u)|=1\right\}=\|\sigma\|,
\end{aligned}
$$

which completes the proof.

Let $\mathscr{F}(\sigma)$ denote the Fourier transform of a measure $\sigma$. We note that $\mathscr{H}$ is a proper subset of $C[0, T]$. Now the following theorem holds.

Theorem 19. For $\sigma \in \mathscr{M}(\mathscr{H})$, let $\mu=\mathscr{D} \sigma \in \mathscr{M}$. Then we have $\mathscr{F}(\sigma)=\mathscr{F}(\mu) \circ D=\left.F^{\mu}\right|_{\mathscr{H}}$, where $\mu$ and $F^{\mu}$ are related by $(20)$.

Proof. Since $\beta^{\prime}$ is continuous on $[0, T]$, we can take $M>0$ such that $\beta^{\prime}(t) \leq M$ for all $t \in[0, T]$. Then it follows that for all $v \in \mathscr{H}$

$$
\int_{0}^{T}\left[v^{\prime}(t)\right]^{2} d \beta(t) \leq M^{2}\|D v\|_{0, \beta}^{2}=M^{2}\|v\|_{\mathscr{H}}^{2}<\infty .
$$

This means that $v^{\prime} \in L_{0, \beta}^{2}[0, T]$ for all $v \in \mathscr{H}$. Thus, by Lemma 1, the integral of the right-hand side of (20) exists; that is, $F^{\mu}(v)$ exists. We now have by the change of variable theorem

$$
\begin{aligned}
& \left.F^{\mu}\right|_{\mathscr{H}}(v) \\
& =\int_{L_{\alpha, \beta}^{2}[0, T]} \exp \left\{i \int_{0}^{T} f(t) v^{\prime}(t) d m_{L}(t)\right\} d \mu(f) \\
& =\int_{L_{\alpha, \beta}^{2}[0, T]} \exp \left\{i \int_{0}^{T} f(t) D v(t) d \beta(t)\right\} d\left(\sigma \circ D^{-1}\right)(f) \\
& =\int_{\mathscr{H}} \exp \left\{i \int_{0}^{T} D u(t) D v(t) d \beta(t)\right\} d \sigma(u) \\
& =\int_{\mathscr{H}} \exp \left\{i\langle u, v\rangle_{\mathscr{H}}\right\} d \sigma(u)=\mathscr{F}(\sigma)(v)
\end{aligned}
$$


and since $L_{\alpha, \beta}^{2}[0, T]=L_{0, \beta}^{2}[0, T]$ as vector spaces with the same Borel $\sigma$-algebra $\mathscr{B}$, we have

$$
\begin{aligned}
& \left.F^{\mu}\right|_{\mathscr{H}}(v) \\
& \quad=\int_{L_{0, \beta}^{2}[0, T]} \exp \left\{i \int_{0}^{T} f(t) D v(t) d \beta(t)\right\} d \mu(f) \\
& \quad=\int_{L_{0, \beta}^{2}[0, T]} \exp \left\{i\langle f, D v\rangle_{0, \beta}\right\} d \mu(f) \\
& \quad=\mathscr{F}(\mu)(D v),
\end{aligned}
$$

which completes the proof.

Let $\mathscr{F}(\mathscr{H})$ be the Fresnel class $[4,15]$ defined by

$$
\mathscr{F}(\mathscr{H})=\{\mathscr{F}(\sigma): \sigma \in \mathscr{M}(\mathscr{H})\} .
$$

Then the correspondence $\sigma \rightarrow \mathscr{F}(\sigma)$ is injective and carries convolution to point-wise multiplication. Letting $\|\mathscr{F}(\sigma)\|=$ $\|\sigma\|$, we have that $\mathscr{M}(\mathscr{H})$ is isometrically isomorphic to $\mathscr{F}(\mathscr{H})$ and $\mathscr{F}(\mathscr{H})$ is a Banach algebra [15]. The Fresnel integral $\mathscr{F}_{I}[\mathscr{F}(\sigma)]$ is defined for $\mathscr{F}(\sigma) \in \mathscr{F}(\mathscr{H})$ by the formula

$$
\mathscr{F}_{I}[\mathscr{F}(\sigma)]=\int_{\mathscr{H}} \exp \left\{-\frac{i}{2}\|h\|_{\mathscr{H}}^{2}\right\} d \sigma(h) .
$$

Now we have the following isomorphism theorem.

Theorem 20. The map $\phi: \mathcal{S}_{\alpha, \beta ; \varphi} \rightarrow \mathscr{F}(\mathscr{H})$ defined by $\phi\left(F^{\mu}\right)=\mathscr{F}(\sigma)=\left.F^{\mu}\right|_{\mathscr{H}}$ for $\mu \in \mathscr{M}$, where $\sigma=\mathscr{I} \mu \in \mathscr{M}(\mathscr{H})$, is an isometric isomorphism as Banach algebras. The inverse $\phi^{-1}$ of $\phi$ is given by $\phi^{-1}[\mathscr{F}(\sigma)]=F^{\mu}$ for $\sigma \in \mathscr{M}(\mathscr{H})$, where $\mu=\mathscr{D} \sigma \in \mathscr{M}$.

Proof. Let $F^{\mu} \in \mathcal{S}_{\alpha, \beta ; \varphi}$ with $\mu \in \mathscr{M}$ and let $\sigma=\mathscr{I} \mu$. By Theorem 13, the map $\phi_{1}: \mathcal{S}_{\alpha, \beta ; \varphi} \rightarrow \mathscr{M}$ defined by $\phi_{1}\left(F^{\mu}\right)=\mu$, is an isometric isomorphism between Banach algebras. By Lemma 18, the map $\mathscr{I}: \mathscr{M} \rightarrow \mathscr{M}(\mathscr{H})$ is also an isometric isomorphism between Banach algebras. Let $\phi_{2}: \mathscr{M}(\mathscr{H}) \rightarrow$ $\mathscr{F}(\mathscr{H})$ be defined by $\phi_{2}(\sigma)=\mathscr{F}(\sigma)$ for $\sigma \in \mathscr{M}(\mathscr{H})$. By Proposition 2.3 of [15], $\phi_{2}$ also is an isometric isomorphism between Banach algebras, too. Now we have $\phi=\phi_{2} \circ \mathscr{I} \circ \phi_{1}$ and this theorem follows by Theorem 19 .

Theorem 21. For $\mu \in \mathscr{M}$ and $F^{\mu} \in \mathcal{S}_{\alpha, \beta ; \varphi}$, let $F^{\mu}$ and $\mu$ be related by (20). Let $\sigma=\mathscr{I} \mu$. Then we have the following:

(1) If $\lambda>0$, then

$$
\begin{gathered}
J_{F}(\lambda)=\int_{L_{\alpha, \beta}^{2}[0, T]} \exp \left\{-\frac{1}{2 \lambda}\|f\|_{0, \beta}^{2}\right. \\
\left.+i \lambda^{-1 / 2} \int_{0}^{T} f(t) d \alpha(t)\right\} d \mu(f) \\
=\int_{\mathscr{H}} \exp \left\{-\frac{1}{2 \lambda}\|v\|_{\mathscr{H}}^{2}\right. \\
\left.+i \lambda^{-1 / 2} \int_{0}^{T} D v(t) d \alpha(t)\right\} d \sigma(v) .
\end{gathered}
$$

(2) If $\lambda>0$ and $\alpha^{\prime} \in L_{\alpha, \beta}^{2}[0, T]$ with $\alpha(0)=0$ (or equivalently $\alpha \in \mathscr{H}$ ), then

$$
J_{F}(\lambda)=\int_{\mathscr{H}} \exp \left\{-\frac{1}{2 \lambda}\|v\|_{\mathscr{H}}^{2}+i \lambda^{-1 / 2}\langle v, \alpha\rangle_{\mathscr{H}}\right\} d \sigma(v) \text {. }
$$

(3) If there exists $M>0$ satisfying

$$
\begin{aligned}
& \int_{L_{\alpha, \beta}^{2}[0, T]} \exp \left\{\operatorname{Re}\left(i \lambda^{-1 / 2}\right) \int_{0}^{T} f(t) d \alpha(t)\right\} d|\mu|(f) \\
& \quad \leq M
\end{aligned}
$$

for any $\lambda \in \mathbb{C}_{+}$, then $E_{w_{\alpha, \beta}, \varphi}^{a n w_{\lambda}}\left[F^{\mu}\right]$ is given by the righthand sides of (60). Moreover, if (62) holds for $\lambda \epsilon$ $\{\lambda \in \mathbb{C}: \operatorname{Re} \lambda \geq 0, \lambda \neq 0\}$, then for any nonzero real $q, E_{w_{\alpha, \beta ;} \varphi}^{a n f_{q}}[F]$ is given by the right-hand sides of (60) replacing $\lambda$ by -iq. In addition, if $\alpha \in \mathscr{H}$, then $E_{w_{\alpha, \beta ; \varphi}}^{a n f_{q}}[F]$ is given by the right-hand side of (61) replacing $\lambda$ by-iq.

Proof. Since $\mu=\mathscr{I}^{-1} \sigma=\mathscr{D} \sigma=\sigma \circ D^{-1}$, we have for $\lambda>0$

$$
\begin{aligned}
J_{F} & (\lambda)=\int_{C[0, T]} F^{\mu}\left(\lambda^{-1 / 2} x\right) d w_{\alpha, \beta ; \varphi}(x) \\
& =\int_{L_{\alpha, \beta}^{2}[0, T]} \int_{C[0, T]} \exp \left\{i \lambda^{-1 / 2} I_{\alpha, \beta}(f)(x)\right\} d w_{\alpha, \beta ; \varphi}(x) d \mu(f) \\
& =\int_{L_{\alpha, \beta}^{2}[0, T]} \exp \left\{-\frac{1}{2 \lambda}\|f\|_{0, \beta}^{2}\right. \\
& \left.+i \lambda^{-1 / 2} \int_{0}^{T} f(t) d \alpha(t)\right\} d\left(\sigma \circ D^{-1}\right)(f) \\
& =\int_{\mathscr{H}} \exp \left\{-\frac{1}{2 \lambda}\|v\|_{\mathscr{H}}^{2}+i \lambda^{-1 / 2} \int_{0}^{T} D v(t) d \alpha(t)\right\} d \sigma(v)
\end{aligned}
$$

by the Fubini theorem and the change of variable theorem, which proves (60). Moreover, it is not difficult to prove that $\alpha^{\prime} \in L_{\alpha, \beta}^{2}[0, T]$ with $\alpha(0)=0$ if and only if $\alpha \in \mathscr{H}$ since $\beta^{\prime}$ is bounded and bounded away from 0 . Now if $\alpha^{\prime} \in L_{\alpha, \beta}^{2}[0, T]$ with $\alpha(0)=0$, then we have

$$
\begin{aligned}
J_{F}(\lambda) & =\int_{\mathscr{H}} \exp \left\{-\frac{1}{2 \lambda}\|v\|_{\mathscr{H}}^{2}\right. \\
& \left.+i \lambda^{-1 / 2} \int_{0}^{T} D v(t) D \alpha(t) d \beta(t)\right\} d \sigma(v) \\
& =\int_{\mathscr{H}} \exp \left\{-\frac{1}{2 \lambda}\|v\|_{\mathscr{H}}^{2}+i \lambda^{-1 / 2}\langle v, \alpha\rangle_{\mathscr{H}}\right\} d \sigma(v),
\end{aligned}
$$

which proves (61). If (62) holds, then we have (3) of this theorem by the definition of the generalized analytic Feynman $w_{\alpha, \beta ; \varphi}$-integral and the dominated convergence theorem.

Remark 22. Applying (3) of Theorem 21, we can also obtain Theorem 3.1 of [2].

Letting $M=\|\mu\|$ in (62) of Theorem 21, we now have the following corollary which is one of our main results. 
Corollary 23. For $\mu \in \mathscr{M}$ and $F^{\mu} \in \mathcal{S}_{\alpha, \beta ; \varphi}$, let $F^{\mu}$ and $\mu$ be related by (20). If $\alpha(t)=0$ for all $t \in[0, T]$, then we have for any $\lambda \in \mathbb{C}_{+}$

$$
\begin{aligned}
E_{w_{\alpha, \beta ; \varphi}}^{a n w_{\lambda}}\left[F^{\mu}\right] & =\int_{L_{\alpha, \beta}^{2}[0, T]} \exp \left\{-\frac{1}{2 \lambda}\|f\|_{0, \beta}^{2}\right\} d \mu(f) \\
& =\int_{\mathscr{H}} \exp \left\{-\frac{1}{2 \lambda}\|v\|_{\mathscr{H}}^{2}\right\} d \sigma(v),
\end{aligned}
$$

where $\sigma=\mathscr{I} \mu$. Moreover, for any nonzero real $q, E_{w_{\alpha, \beta ; \varphi}}^{a n f_{q}}[F]$ is given by the right-hand sides of (65) replacing $\lambda$ by-iq. In this case, we have $E_{w_{\alpha, \beta ; \varphi}}^{a n f_{1}}\left[F^{\mu}\right]=\mathscr{F}_{I}[\mathscr{F}(\sigma)]$.

\section{Data Availability}

No data were used to support this study.

\section{Conflicts of Interest}

The author declares no conflicts of interest.

\section{Acknowledgments}

This research was supported by the Basic Science Research Program through the National Research Foundation (NRF) of Korea funded by the Ministry of Education (2017R1D1A1B03029876).

\section{References}

[1] R. H. Cameron and D. A. Storvick, "Some Banach algebras of analytic Feynman integrable functionals," in Analytic Functions Kozubnik 1979, vol. 798 of Lecture Notes in Mathematics, pp. 1867, Springer, Berlin, Germany, 1980.

[2] S. J. Chang and J. G. Choi, "Effect of drift of the generalized Brownian motion process: an example for the analytic Feynman integral," Archiv der Mathematik, vol. 106, no. 6, pp. 591-600, 2016.

[3] S. J. Chang and D. Skoug, "Generalized Fourier-Feynman transforms and a first variation on function space," Integral Transforms and Special Functions, vol. 14, no. 5, pp. 375-393, 2003.

[4] G. W. Johnson, "The equivalence of two approaches to the Feynman integral," Journal of Mathematical Physics, vol. 23, no. 11, pp. 2090-2096, 1982.

[5] S. A. Albeverio and R. J. Høegh-Krohn, "Mathematical theory of Feynman path integrals," in Lecture Notes in Mathematics, Springer-Verlag, Berlin, Germany, 1976.

[6] K. S. Chang, G. W. Johnson, and D. L. Skoug, "Functions in the Fresnel class," Proceedings of the American Mathematical Society, vol. 100, no. 2, pp. 309-318, 1987.

[7] S. J. Chang, J. G. Choi, and S. D. Lee, "A Fresnel type class on function space," Journal of the Korean Society of Mathematical Education. Series B. The Pure and Applied Mathematics, vol. 16, no. 1, pp. 107-119, 2009.

[8] K. S. Ryu, "The generalized analogue of Wiener measure space and its properties," Honam Mathematical Journal, vol. 32, no. 4, pp. 633-642, 2010.
[9] K. S. Ryu, "The translation theorem on the generalized analogue of Wiener space and its applications," Journal of the Chungcheong Mathematical Society, vol. 26, no. 4, pp. 735-742, 2013.

[10] D. H. Cho, "Measurable functions similar to the Itô integral and the Paley-Wiener-Zygmund integral over continuous paths," Preparation, In Press.

[11] I. D. Pierce, On a family of generalized Wiener spaces and applications [Ph.D. thesis], University of Nebraska-Lincoln, Lincoln, Neb, USA, 2011.

[12] E. Hewitt and K. Stromberg, Real and abstract analysis, Springer-Verlag, New York-Heidelberg, 1975.

[13] H. H. Kuo, "Gaussian measures in Banach spaces," in Lecture Notes in Mathematics, Springer-Verlag, Berlin, Germany, 1975.

[14] I. Yoo, B. J. Kim, and B. S. Kim, "A change of scale formula for a function space integral on $C_{a, b}[0, T]$," Proceedings of the American Mathematical Society, vol. 141, no. 8, pp. 2729-2739, 2013.

[15] G. Kallianpur and C. Bromley, "Generalized Feynman integrals using analytic continuation in several complex variables," in Stochastic Analysis and Applications, vol. 7 of Advanced Probability and Related Topics, pp. 217-267, Dekker, NY, USA, 1984. 


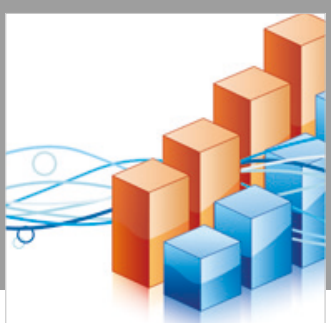

Advances in

Operations Research

\section{-n-m}
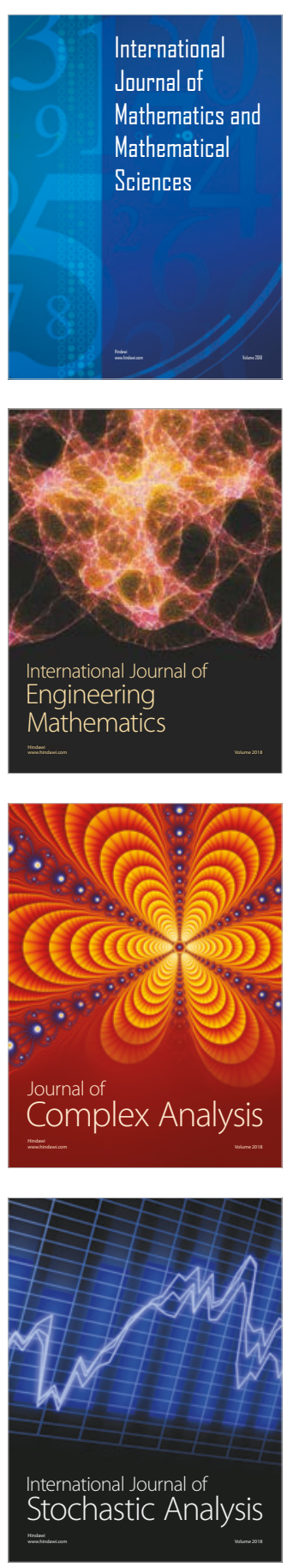
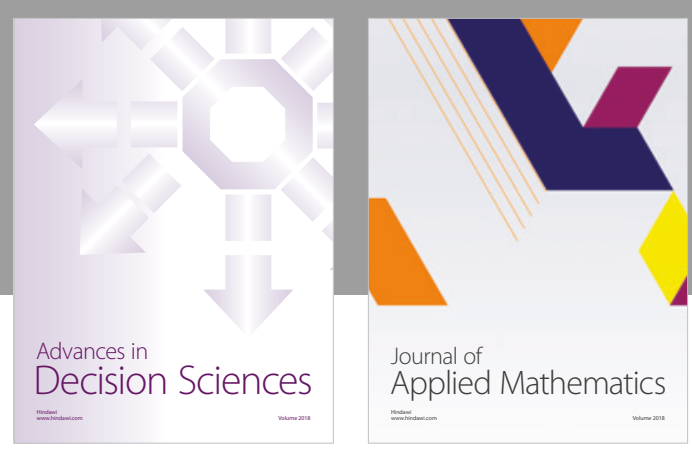

Journal of

Applied Mathematics
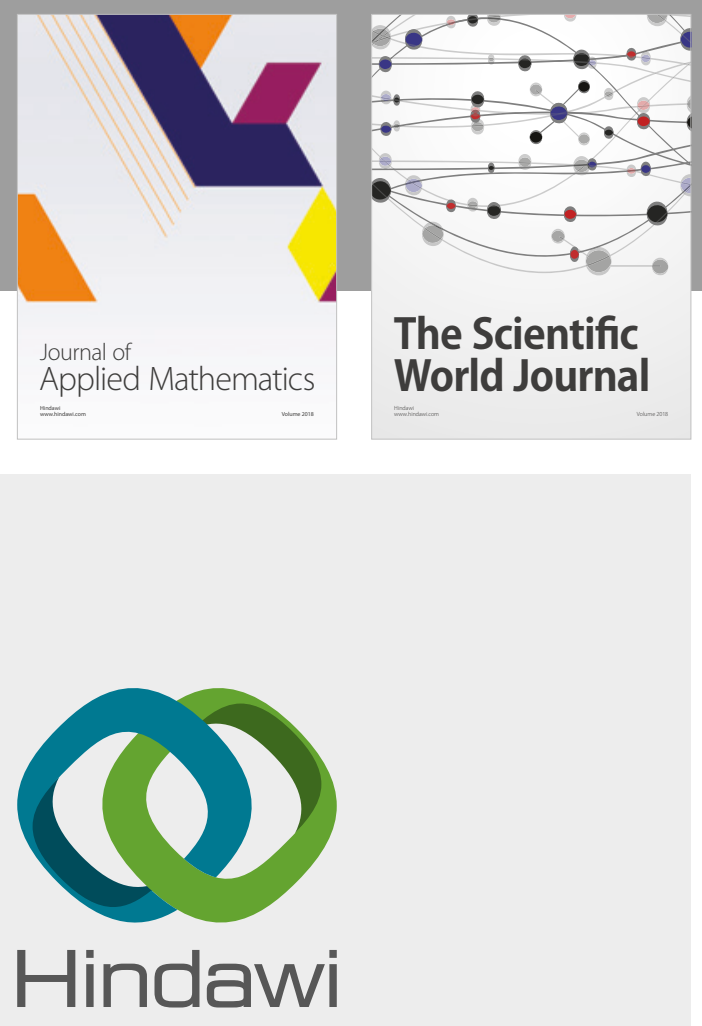

Submit your manuscripts at

www.hindawi.com

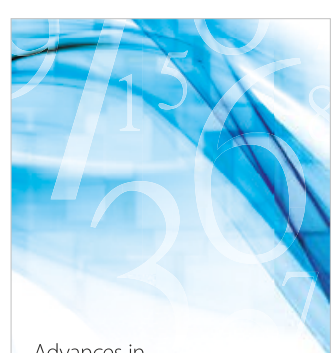

Advances in
Numerical Analysis
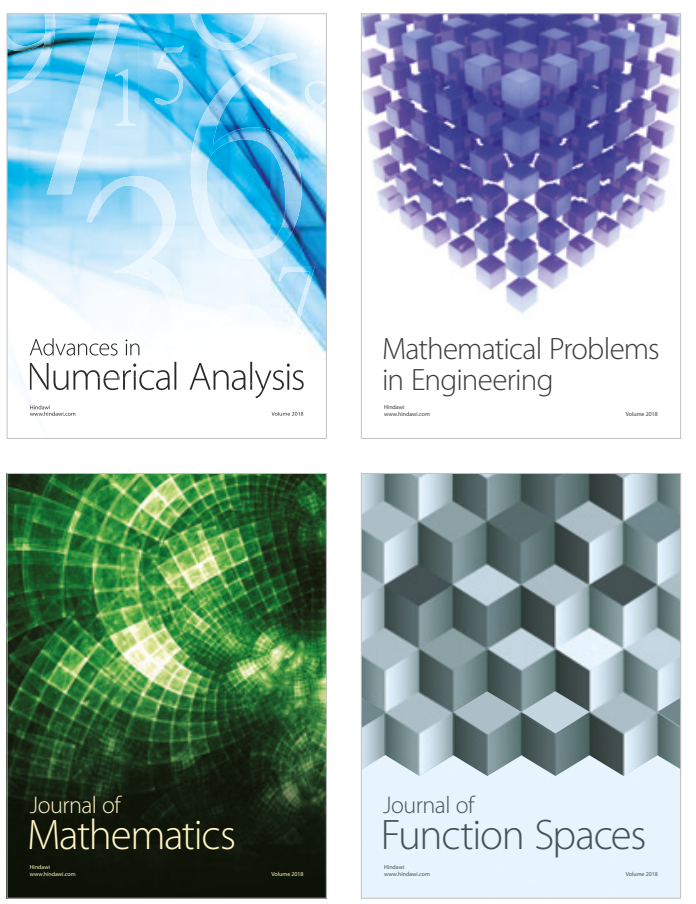

Mathematical Problems in Engineering

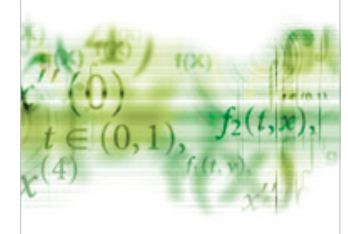

International Journal of

Differential Equations

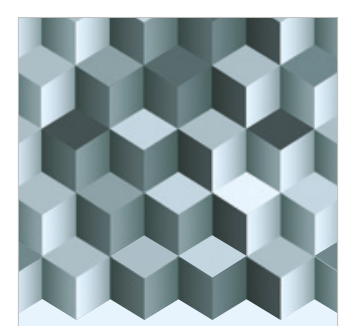

Journal of

Function Spaces

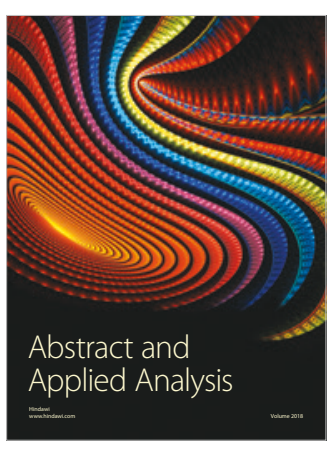

The Scientific

World Journal

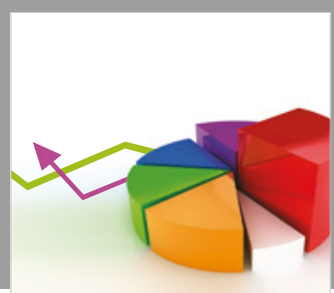

Journal of

Probability and Statistics
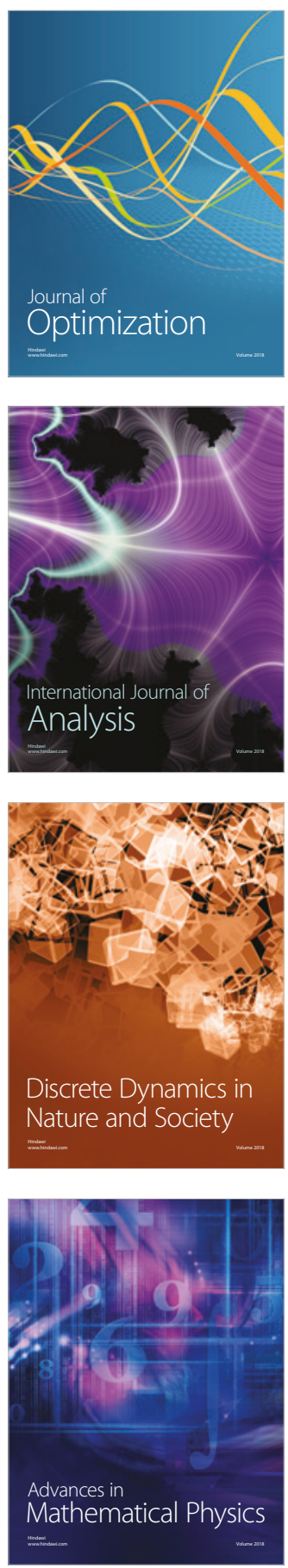